\title{
POUR UNE CONCEPTION HISTORICISÉE ET GRADUELLE DE LA POÉTICITÉ. L'INTERACTION DES DIMENSIONS SÉMANTIQUE, ÉNONCIATIVE ET ICONIQUE DANS TROIS POÈMES (SPONDE, FOLLAIN, BONNEFOY) / FOR A HISTORICAL AND GRADUAL CONCEPTION OF POETRY. THE INTERACTION OF SEMANTIC, ENUNCIATIVE AND ICONIC DIMENSIONS IN THREE POEMS (SPONDE, FOLLAIN, BONNEFOY)
}

Résumé: Nous soutenons dans cet article l'idée que les textes poétiques, comme les autres textes, devraient être analysés selon 3 perspectives: sémantique, iconique et énonciative, chacune possédant un pôle positif et un pôle négatif. Du point de vue sémantique, les textes poétiques et scientifiques sont tous deux sur le pôle positif, parce qu'ils créent une nouvelle représentation du monde. $\mathrm{Du}$ point de vue iconique, ce que Jakobson appelait la fonction poétique, les poèmes se situent généralement près du pôle positif mais leur position exacte dépend de caractéristiques formelles qui ont changé au fil du temps. Ils partagent cette position avec des textes tels que les publicités, les chansons et les comptines. Du point de vue énonciatif, beaucoup de textes poétiques appartenant au genre lyrique occupent une position moyenne entre les deux pôles, du fait qu'ils ne

\footnotetext{
Professora da Universidade de Toulon, França; michele.monte@univ-tln.fr
} 
montrent pas leur nature littéraire et se donnent pour des énoncés de la vie ordinaire, mais il en va différemment pour l'épopée, qui se situe près du pôle positif, comme les romans et les autres textes fictionnels. En définissant 3 perspectives différentes et en situant chaque texte par rapport à un pôle négatif et un pôle positif, nous pouvons mieux apprécier la spécificité des textes poétiques au sein des autres textes, mais aussi prendre en compte l'évolution très forte de la poésie au fil des siècles. Après avoir présenté notre modèle, nous le mettons à l'épreuve de trois poèmes très différents les uns des autres : un sonnet baroque de Jean de Sponde, un poème en vers libres de Jean Follain et un poème en prose d'Yves Bonnefoy.

Mots-clé: poésie, textualité, vers et prose, décontextualisation, parallélismes

Abstract: We argue in this paper that poetic texts, as well as any other texts should be analysed through 3 perspectives: semantic, iconic and enunciative, each one with a positive pole and a negative one. According to the semantic perspective, poetic and scientific texts are both on the positive pole, because they create a new representation of the world. According to the iconic one, that Jakobson called the poetic function, poems are generally near the positive pole but their accurate position depends on formal characteristics which have changed along history. They share this position with texts such as advertisements, songs or nursery rhymes. According to enunciation, a lot of poetical texts belonging to lyrics stand on an average position between the 2 poles, since they do not show their literary origin and give themselves as ordinary utterances, but it is not the case for epics, which stands very near from the positive pole, such as novel and fictional texts. Defining 3 different perspectives and ranking texts from a positive to a negative pole for each of them allow to better appreciate the specificity of poetical texts 
among other texts but also to take into account the very important evolution of poetry along the centuries. After presenting our model, we make it work on 3 different poems: a baroque sonnet from Jean de Sponde, a free-verse poem from Jean Follain and a prose poem from Yves Bonnefoy.

Keywords: poetry, textuality, verse and prose, decontextualization, parallels

\section{Introduction}

J'ai défendu dans ma contribution au Congrès Mondial de Linguistique Française de $2012^{1}$ une approche du texte poétique qui, sans rien renier des apports fondamentaux de grands linguistes tels que Jakobson, Ruwet, Benveniste ou Dominicy, prend en compte la complexité du texte poétique en faisant l'hypothèse d'une autonomie relative de trois perspectives que j'ai qualifiées de sémantique, iconique et énonciative. Après avoir résumé mes propositions, j'essaierai de voir sur trois exemples en quoi elles peuvent permettre de mieux appréhender le fonctionnement linguistique du texte poétique. J'étudierai un sonnet baroque, un poème en vers libres et un poème en prose ${ }^{2}$.

\section{Trois niveaux d'appréhension du texte poétique}

Il me semble tout d'abord important de rappeler qu' on ne saurait opposer, comme le faisait Jean Cohen en son temps, la langue poétique et la langue ordinaire. La première raison est que la langue ordinaire n'existe pas, il n'y a que des usages spécifiques du langage, façonnés par des pratiques sociales ayant donné naissance à des genres en perpétuel remodèlement, dont certains sont appelés par l'évolution de la vie sociale à disparaître et d'autres à émerger à partir de modèles antérieurs, comme on le voit à l'heure actuelle avec les blogs

1 Este artigo, originalmente, publicado em Revue Recherches: Langue et discours. Actes du XVIIIe Séminaire de Didactique Universitaire Constanta, 2011. Cluj: Echinox, 2012, p. 14-39.

2 J'applique les modifications orthographiques recommandées par l'Académie française (1990). 
et les forums de discussion sur Internet. La deuxième raison est qu'il vaut mieux envisager la poéticité non pas à partir d'une improbable langue poétique mais à partir des textes poétiques eux-mêmes, qu'il faudra situer, d'une part, par rapport à d'autres textes littéraires non poétiques, d'autre part, par rapport à des genres non littéraires avec lesquels ils sont en fréquent dialogue. Pour prendre rapidement un exemple, pensons à deux genres poétiques majeurs et d'une grande permanence historique, l'ode et l'élégie ${ }^{3}$ : ces deux genres s'enracinent, pour l'ode dans des pratiques discursives liées à la célébration de la cité ou des hommes illustres qui l'honorent ou à la commémoration d'évènements collectifs importants tels qu'une victoire ou la naissance d'un héritier du trône royal, et pour l'élégie à la déploration mêlée d'éloge occasionnée par la perte d'un être aimé ou, plus largement, à un sentiment de nostalgie liée à une idéalisation du passé. C'est par rapport à ces pratiques discursives non littéraires que l'on peut situer ces deux genres, tout en prenant en compte également l'histoire propre du genre poétique et notamment la différenciation dans la poétique classique entre le style sublime et le style tempéré. C'est de cette façon que l'on pourra le mieux appréhender la spécificité de poèmes tels que les Odas elementales de Pablo Neruda ou Couur élégie rouge de James Sacré, en mesurant la façon dont ils assument et/ou contestent la tradition.

Après ce préalable méthodologique, je propose de considérer les textes, et pas seulement les textes poétiques, sous trois aspects partiellement indépendants. Un texte se caractérise tout d'abord par une certaine représentation discursive qui met en mots le réel en s'appuyant sur des dénominations et des schématisations (Grize 1999) partagées dans la communauté discursive et en en créant éventuellement de nouvelles. Moirand (2007) a bien montré par exemple à propos des crises sanitaires (sang contaminé, vache folle, OGM) comment les discours de presse parlaient d'évènements nouveaux en les intégrant à des paradigmes anciens, jouant ainsi un rôle de passeur entre les spécialistes du monde médical et les lecteurs. Sous cet angle que j'appelle sémantique, les textes poétiques comme les textes scientifiques mais par des moyens différents modifient nos représentations, introduisent de nouvelles schématisations, moins stables dans nos cultures modernes que celles qui sont produites par les

\footnotetext{
Concernant l'élégie, je me permets de renvoyer à l'introduction du numéro de la revue Babel intitulé Élégies quej'ai coordonné et que l'on peut se procurer auprès de la Faculté de Lettres de Toulon (Monte 2005).
} 
scientifiques et diffusées ensuite par l'enseignement, mais qui peuvent malgré tout affecter durablement nos manières de dire et de penser. On peut penser à cet égard aux poèmes des troubadours qui ont imprégné profondément la façon de penser les relations amoureuses. L'étude sémantique d'un poème s'attachera ainsi à observer la représentation du monde qui y est proposée, les éventuelles anomalies par rapport à des visions plus standardisées, anomalies dont elle pourra rendre compte en termes de ruptures d'isotopies (Rastier 1987) et de pragmatique des figures (Bonhomme 2005) mais aussi de discontinuité textuelle ou de flottement référentiel. L'interaction avec le niveau iconique que je vais définir ci-dessous peut créer à l'inverse une solidarité sémantique forte entre lexèmes d'ordinaire dissociés et créer de nouvelles isotopies.

Un texte est par ailleurs le produit de la mise en œuvre d'un certain matériau verbal, et il peut dans sa texture même attirer l'attention sur ce matériau ou au contraire le rendre en quelque sorte transparent au profit de ce dont il est question dans le texte. On opposera ainsi les textes qui subordonnent l'emploi du matériau verbal à la visée sémantique et ceux qui s'appuient sur le matériau verbal pour produire des effets sémantiques et esthétiques. Sur ce versant iconique du texte, on trouvera d'un côté les textes scientifiques, didactiques, informatifs, qui, sauf exception ${ }^{4}$, n'ont qu'une approche utilitaire du matériau verbal, et de l'autre, les textes poétiques qui tirent une grande partie de leur efficace du jeu des composantes phoniques et syntactico-rythmiques ainsi que de la disposition typographique (ce que Michel Favriaud a appelé la ponctuation blanche). Entre ces deux pôles on placera tous les textes qui accordent de l'importance à ces facteurs mais en les subordonnant à une finalité persuasive ou ludique - textes publicitaires, textes d'éloquence, jeux de mots - ou à la création d'un monde fictionnel - littérature narrative et dramatique -. Si je choisis pour cette dimension du texte le terme iconique plutôt que poétique, c'est parce que je voudrais réserver le terme poétique uniquement à une certaine catégorie de textes littéraires reconnus par les locuteurs comme ayant des propriétés spécifiques mais variables selon les époques et les cultures. Quant au terme iconique, il dérive bien sûr de l'emploi du mot icône dans la sémiotique de Peirce. Le terme iconicité a été introduit par Jakobson (1966) qui l'oppose à l'arbitraire du signe tel que défini par Saussure. Le choix de ce mot qui insiste sur la relation de similitude entre la

${ }^{4}$ Les titres, notamment dans les médias, sont souvent le lieu d'une recherche qui inclut la dimension iconique du langage. 
structuration du matériau verbal et le sens des énoncés découle de l'analyse des parallélismes poétiques proposée par Ruwet (1975) et approfondie par Dominicy (2011). On sait que le texte poétique se caractérise dans sa texture par une double structuration: la structuration logico-grammaticale habituelle du langage et la structuration par les parallélismes qui affectent à la fois les phonèmes, la répartition des accents, la syntaxe, et parfois la disposition spatiale. Ruwet montre que la seconde agit en retour sur la première, soit en palliant des incohérences ou du flou dus à un défaut de structuration, soit en conduisant le récepteur à inférer de nouveaux liens sémantiques entre lexèmes ou énoncés ainsi mis en parallèle. Dominicy défend l'idée selon laquelle ce sont les parallélismes qui déclenchent chez le récepteur un traitement spécifique du contenu sémantique du poème qu'il appelle l'évocation :

la structure double du poème donne lieu à deux processus évocatifs qui se déroulent en tandem : l'un a pour input les percepts inanalysés que suscite l'organisation poétique ; l'autre, les concepts inanalysés que renferment les contenus propositionnels extraits de l'organisation linguistique $(2011: 245)^{5}$.

Cette conception de l'iconicité, ainsi que Dominicy le montre aux p.88106 de son livre, s'écarte de celle qui attribuerait une valeur symbolique ou synesthésique directe aux sons et aux mélodies. En effet, les effets sonores sont grandement dépendants de l'organisation linguistique (frontières de mots et de groupes) et métrique (frontières de vers ou d'hémistiches). C'est pourquoi Dominicy critique la mise en cause de l'arbitraire du signe par Jakobson 1966. Si donc il y a bel et bien une iconicité du texte poétique, j'entendrai le mot non pas comme l'attribution d'une motivation directe aux éléments non significatifs du langage, mais comme le fait que le texte attire l'attention du récepteur sur son matériau et ce faisant oriente, si l'on adopte l'hypothèse de Dominicy, la construction de la signification vers la réactivation de souvenirs engrangés dans la mémoire personnelle plus que vers des propositions dotées d'une vérité sémantique universelle. Les progrès actuels dans la compréhension des mécanismes de la mémoire et de la construction des représentations discursives bénéficieront sans doute à l'analyse du rôle du matériau verbal dans la production

Les termes « percept 》 et « concept inanalysé » sont définis précisément par Dominicy et s'appuient sur une vision cognitive du fonctionnement du langage que je ne peux développer ici. 
de la signification, les travaux sémiotiques sur d'autres matériaux tels que les formes, les couleurs et les sons pourront aussi nous éclairer sur ces processus qui ne s'appuient pas sur l'agencement des concepts. Pour ma part, je me bornerai à observer comment les parallélismes phoniques, métriques, syntaxiques interagissent avec l'organisation linguistique, soit en la redoublant, soit en introduisant des écarts auxquels le récepteur attribue une signification.

Un texte, enfin, peut être appréhendé sous l'angle de son fonctionnement énonciatif. Plusieurs typologies énonciatives existent déjà qui ont toutes leur intérêt dans l'approche des textes. Je songe notamment à l'actualisation de la subjectivité dans la théorie praxématique (Barbéris 2001), à la typologie quadripartite proposée par Bronckart (1996) ou aux réflexions sur l'effacement énonciatif proposées par Vion (2001). Mais ce que je vise ici par l'axe énonciatif est antérieur à ces caractérisations. Je vise en effet à opposer les textes dont la scène d'énonciation est donnée comme explicitement distincte de la situation de communication et ceux qui feignent de les confondre dans l'illusion d'une coïncidence parfaite entre l'être-du-monde, pour reprendre les termes de Ducrot (1984 : 199), et le locuteur construit par le texte. L'écart entre le je empirique et le locuteur est théorisé en argumentation par l'opposition entre l'éthos préalable et l'éthos discursif (Amossy 2000 : 70), de même que l'écart symétrique entre l'auditoire réel et l'auditoire construit par le texte (ibid. : 36-37), mais il est contre-intuitif et rarement pris en compte en dehors des spécialistes d'analyse du discours. Bronckart est sensible à la dimension spécifique du je et du nous décontextualisés du discours théorique mais il n'en fait pas un élément pivot de sa classification. Or il me semble que, sur cet axe, on peut trouver à un pôle tous les textes qui ne questionnent pas le processus d'émergence dans et par la prise de parole d'une scène énonciative distincte de la réalité non verbale et à l'autre pôle ceux qui, par leur fictionnalité, exhibent cet écart en donnant la parole à des énonciateurs dont on sait qu'ils ne sont pas les producteurs empiriques du discours, et en créant un monde référentiel dont le caractère complètement ou partiellement imaginaire est reconnu par le lecteur.

On pourrait peut-être penser que cette opposition reconduit la distinction entre textes littéraires et non littéraires, mais ce serait ne pas tenir compte d'une part de l'existence de textes autres que littéraires qui stipulent cette dissociation entre sujet empirique et locuteur - blagues et devinettes à la première personne, certaines scénographies publicitaires -, d'autre part, de l'hétérogénéité des textes littéraires. Comme l'a bien montré Genette (1991), la fiction ne concerne qu'un 
pan de la littérature. Lui-même propose (p.32) de distinguer deux régimes de littérarité : la littérarité constitutive et la littérarité conditionnelle, cette dernière concernant des textes tels que les mémoires, les journaux intimes, les autobiographies, les textes historiques ou philosophiques, qui peuvent ou non être reconnus comme littéraires selon qu'ils possèdent ou pas des qualités particulières au niveau de leur elocutio. Quant à la littérarité constitutive, elle concerne des textes qui sont d'emblée étiquetés comme littéraires, indépendamment de la qualité de leur elocutio, les uns parce qu'ils sont fictionnels, les autres parce qu'ils possèdent ces fameux parallélismes dont je viens de parler ${ }^{6}$. Je garde l'opposition genettienne fiction / diction mais en la déplaçant quelque peu puisque, pour moi, la fiction a trait avant tout aux instances énonciatives et seulement par corollaire au monde représenté. Sur l'axe que je propose, les textes fictionnels (roman, théâtre) se trouvent à un des pôles, mais les textes littéraires non fictionnels, qu'il s'agisse de poésie lyrique ou de littérarité conditionnelle, se trouvent sur une position intermédiaire : ils donnent en effet à entendre que leur locuteur n'est pas exactement assimilable au sujet empirique même s'ils ne font pas de cet écart un des pivots de leur fonctionnement. Dans les autobiographies ou les journaux à visée littéraire, l'écart entre ce que Lejeune (1975) appelle le je narrant et le je narré est souvent thématisé et dans la poésie lyrique, fréquentes sont les scènes d'énonciation non réalistes où le locuteur interpelle des êtres inanimés, des morts ou des entités abstraites. Cependant la poésie à la première personne tire souvent un de ses plus gros effets à réception d'une apparente transparence énonciative qui semble abolir la distance entre le locuteur et le sujet empirique, permettant ainsi l'appropriation du texte par son récepteur invité à le réénoncer pour son propre compte, alors même que le travail sur la représentation discursive et sur la dimension iconique du langage écarte le poème des textes ordinaires. D'où, sans doute, la possibilité de réceptions très contrastées de ces textes poétiques, certains lecteurs étant plus sensibles à leur dimension d'objet d'art tandis que d'autres, à la suite de Hamburger (1986) et des théoriciens allemands du je lyrique les entendent comme des énoncés de réalité ».

J'ai montré de façon théorique dans la contribution du CMLF l'intérêt de ce modèle dont les trois axes sont partiellement indépendants et permettent

6 Notons que certains textes cumulent les deux critères de littérarité: c'est le cas, par exemple, des épopées ou de la tragédie classique française. 
tous une application graduelle et non pas dichotomique des critères. Je voudrais ici en faire l'expérience sur trois poèmes aux caractéristiques différentes dont je me propose d'apprécier la poéticité.

\section{Trois régimes de poéticité}

J'ai choisi de travailler sur un sonnet sans titre de Jean de Sponde (1557$1595)^{7}$ appartenant à ses Poèmes chrétiens, un poème de Jean Follain intitulé « Au pays » extrait de Territoires $(1953)^{8}$ et le dernier poème de la suite Derniers raisins de Zeuxis d'Yves Bonnefoy ${ }^{9}$ dont l'édition originale date de 1993 et qui appartient au recueil La Vie errante publié pour la première fois dans son état actuel en 1993. L'appartenance de ces textes à la poésie ne fait pas de doute ni du point de vue de leur auteur (comme le montrent les indications génériques figurant dans le paratexte ou dans leur bibliographie), ni du point de vue de la réception critique : Sponde, Follain, Bonnefoy sont considérés avant tout comme des poètes. Néanmoins les poèmes de Follain et Bonnefoy risquent de susciter la perplexité de certains lecteurs, comme nous le verrons plus loin. Je me propose de les envisager sous les trois dimensions que j'ai proposées en 1 en précisant leur place sur chacun des axes.

\subsection{Un sonnet baroque}

Le poème de Sponde est le sixième d'une série de 12 sonnets. Du point de vue sémantique, il présente une opposition entre la pensée que le locuteur attribue aux autres Hommes et la sienne propre: les Hommes se plaignent en général que leur vie est trop courte, alors que le locuteur souhaite quitter la vie terrestre pour les « beaux séjours » « au prix de qui ce Temps ne monte qu'un moment ». Partant d'un constat banal - « Tout le monde se plaint de la cruelle envie / Que la Nature porte aux longueurs de nos jours »-, le locuteur construit

7 Je cite le texte dans l'édition des Euvres littéraires établie par A. Boase, Genève, Droz, 1978.

8 Il figure à la page 112 dans l'édition Poésie / Gallimard de 1969 qui regroupe Exister et Territoires avec une préface d'Henri Thomas.

9 Il figure à la page 89 dans l'édition Poésie / Gallimard de 1999. 
peu à peu un réseau d'oppositions qui dévalorise le jour et le Temps présents au profit d'une vie après la mort qui est saisie métonymiquement comme un beau séjour « Où séjourne des Temps l'entresuite infinie ». La schématisation proposée n'a rien d'énigmatique mais elle procède par déplacements et requalifications: la mort n'est jamais nommée, la vie présente est qualifiée de « fâcheux détours », le jour n'est qu'un « ombrage sombre » " où le monde s'aveugle et prend son passe-temps ». Sur l'axe sémantique, on peut situer le poème dans une position moyenne: il s'éloigne des discours quotidiens par les requalifications opérées, mais il est très proche du discours des prédicateurs chrétiens de l'époque. La coïncidence des frontières métriques et des groupes syntaxiques propose une lecture sans surprise où les fins de vers et de strophes soulignent les articulations logiques, à une exception près sur laquelle je reviendrai.

Sur le plan énonciatif, la scénographie subit un glissement très intéressant : au début, on est plutôt dans la scénographie du sermon où le locuteur interpelle son auditoire : « Hommes, vous vous trompez ». Le deuxième quatrain instaure un discours cité fictif qui serait celui d'un Homme vraiment convaincu par la prédication chrétienne. Ce discours est ensuite assumé et amplifié par le locuteur premier dans les tercets mais avec un changement remarquable d'interlocuteur puisque ce sont les «beaux séjours » qui font l'objet d'une apostrophe à l'ouverture du premier tercet et c'est à eux qu'il déclare « vous êtes mon désir ». On observe le processus en boucle sur lequel insiste Maingueneau (2007: 62):

La scénographie est ainsi à la fois ce dont vient le discours et ce qu'engendre le discours; elle légitime un énoncé qui, en retour, doit la légitimer, doit établir que cette scénographie dont vient la parole est précisément la scénographie requise pour énoncer comme il convient, selon le cas, la politique, la philosophie, la science, ou pour promouvoir telle marchandise...» (ibid.)

Ce changement énonciatif illustre le mouvement du locuteur qui se détourne des Hommes pour se tourner déjà vers la vie future, il contribue de ce fait à l'iconicité du texte. Or c'est sur cet axe que le texte occupe une position plus marquée du côté du pôle positif : la construction du sonnet accentue fortement sa dimension argumentative, en justifiant les équivalences sémantiques par des phénomènes de redondance et d'antithèse très marqués et de plus en plus denses au fil du texte. Les vers 3 et 4 sont remarquables par la répétition des phonèmes $/ \mathrm{tr} /, / \mathrm{v} /$ et $/ \mathrm{p} /$, les vers 6 et 7 opposent par le sens et la métrique 
« ces fâcheux détours » et « ces plus beaux séjours », et « dépêtrer de » et « revole à », tandis que le vers 8 place «des Temps » à la césure en les associant à « infinie ». Mais ce sont surtout les tercets qui, par les parallélismes syntaxiques, les récurrences des sonorités, le placement des mots clés aux places métriques fortes, produisent la dévalorisation de la vie présente ${ }^{10}$ et l'exaltation des « beaux séjours » pourtant moins présents sur un plan strictement quantitatif. Le plus notable est l'enjambement suivi de ponctuation forte qui caractérise le premier hémistiche du vers 12: occupant une place centrale dans l'ensemble formé par les deux tercets, cet hémistiche résume la position du locuteur et montre par l'enjambement la victoire de l'aspiration à l'éternité sur l'attachement au moment présent. Ce faisant, l'argumentation par le logos s'efface derrière un effet d'évidence qui associe volonté de persuasion et esthétique à l'instar de ce qui se passe dans les messages publicitaires.

On pourrait schématiser ainsi la position de ce poème sur les trois axes que je propose de considérer :

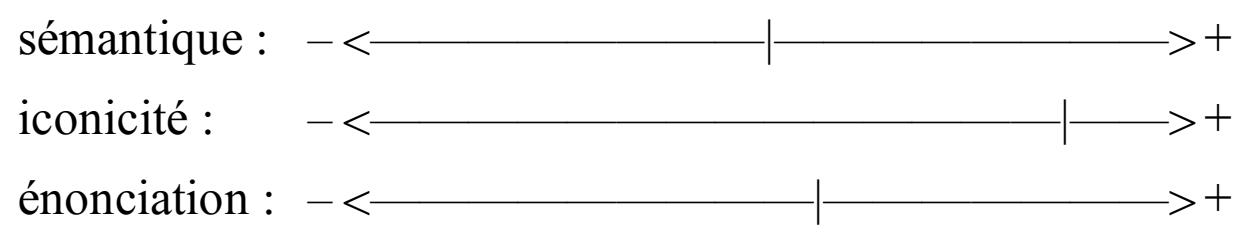

Les lecteurs qui privilégient la nouveauté sémantique comme critère de poéticité trouveront ce poème moins poétique que d'autres mais ceux qui sont sensibles à la densité des parallélismes et à la construction du sonnet caractérisé ici par une pointe qui ramasse toute la leçon du texte lui attribueront un fort coefficient de poéticité. Quant au dispositif énonciatif, il indique par l'apostrophe fictive la construction que représente tout discours par rapport à sa situation matérielle de production / réception mais sans que cela soit fortement souligné: la littérarité du texte est patente sans être pour autant thématisée.

\footnotetext{
${ }^{10}$ On suit la chaine « Temps - moment - jour - ombrage sombre - jour - Temps s'aveugle - passe-temps - moment - Ombre » caractérisée par les voyelles centrales ou postérieures $/ \tilde{\mathrm{a}} /, / \tilde{\mathrm{o}} /, / \mathrm{u} /, / \mathrm{a} /, / \mathrm{e} /, / \mathrm{œP} /$ et les consonnes $/ \mathrm{m} /, / \mathrm{s} /$ et $/ \mathrm{bR} /$, alors que les «beaux séjours » dont les phonèmes sont presque identiques sont différenciés par leur association à la voyelle antérieure /i/ et la consonne /z/ - « au prix de qui », « vous êtes mon désir».
} 


\subsection{Un antipoème?}

Jean Follain (1903-1971) fait partie de ces poètes qui ont commencé à écrire dans l'entre-deux-guerres en se démarquant de l'aventure surréaliste. Sa poésie peut être qualifiée de poésie du quotidien, elle accorde une grande importance aux choses dont Follain fait souvent des sujets actifs qui nous font signe.

Le poème «Au pays » présente plusieurs anomalies ou incertitudes sémantiques : il commence par un « ils » et l'identité des référents du pronom n'est jamais élucidée. On ne connaitra d'eux que cette décision « de s'en aller / au pays ». L'isolement de « au pays » dans le titre et dans le vers 2 crée une incertitude sur ce pays : doit-on construire son sens uniquement par le moyen des relatives qui suivent (v.3-8) interprétées comme déterminatives, ou doit-on plutôt l'entendre comme le pays de ces personnes non identifiées, l'article ayant alors une valeur de notoriété et le pays désignant l'endroit où on est né ${ }^{11}$ ? Le titre nous fait pencher vers la deuxième interprétation, dans laquelle les relatives n'ont plus qu'une valeur descriptive. Mais l'indétermination syntaxique est créatrice de sens : ce pays n'est objet de nostalgie que parce qu'il est conservé dans la mémoire tel que les relatives nous l'évoquent. Le choix exclusif d'articles définis pour actualiser les syntagmes nominaux du poème introduit aussi une incertitude : le SN peut référer à une occurrence précise, inscrite dans le souvenir des « ils », mais aussi à ce que Culioli appelle le type, c'est-à-dire le centre organisateur de la notion représentée par le nom, quand « ce centre correspond à une représentation que l'on peut exhiber ou pointer (objets ; conduites), d'un côté, et par ailleurs, sert de norme, d'étalon, bref de critère de conformité »" (1999 : 71). Le fait qu'il n'y ait pas eu fléchage ou identification anaphorique des SN (Culioli 1999 : 47) nous oriente vers une interprétation en termes de type sans exclure complètement celle en termes d'occurrence. On peut faire l'hypothèse que le choix des définis correspond à un processus selon lequel, dans le souvenir des « ils », des occurrences précises de « mère », de « vieille femme », de « fillette » se sont transformées en type idéalisé. Mais l'intérêt de la poésie est que ce phénomène de typification est inféré sans être explicité.

Le texte est plus bizarre dans l'usage qu'il fait de puis : cet organisateur textuel, temporel ou logique, doit être rapporté à un point de vue sur la situation

${ }^{11}$ C'est le sens qui s'actualise dans l'expression "le mal du pays" ou dans le proverbe « Nul n'est prophète en son pays ». 
représentée. Or, ici aussi, deux possibilités s'offrent à nous : soit il s'agit d'une troisième caractéristique du pays - puis ferait alors série avec les deux où et organiserait l'afflux des souvenirs -, soit il s'agit d'une évolution temporelle de l'enfant devenue fillette, marquant un passage de la petite enfance à la période des amitiés. Au lieu d'ordonner sans heurts une série d'informations, puis, du fait de cette ambigüité, apparaît comme une pierre d'achoppement qui interrompt la linéarité, comme le signale la majuscule à l'initiale. Il sépare le souvenir en deux zones distinctes et correspond aussi à une émancipation du pays par rapport à la décision des « ils ». Mais au moment où la syntaxe semble imposer une représentation du pays indépendante du souvenir, le temps se brouille : le moment du jeu s'étend aux dimensions de toute une vie, celle des « ils » vieillissants décidant de revenir au pays, avec un bouclage du poème revenant à son début. Cette interprétation de la deuxième phrase du poème découle du passage entre tirets « - ô longue vie - » qui amène à penser que le jeu où il s'agit de trouver un objet en s'aidant des indications de l'autre joueur est ici une métaphore de la vie elle-même. Dans cette perspective, l'obscurité des feuillages, à la fin du texte, renverrait non pas seulement à la tombée de la nuit, que les enfants prises par leur jeu n'ont pas vu arriver, mais aussi à la fin de la vie. L'apparente contradiction entre « si longtemps / si tard » et «bientôt » se résout, quant à elle, par un changement de point de vue : il est objectivement tard, mais pour les enfants ou pour l'être humain arrivé « à la fin des fins », c'est arrivé très/ trop vite. Ce dernier vers renouvellerait ainsi le rapport d'analogie entre journée et vie humaine par l'introduction d'un élément très concret renvoyant à des sensations que le lecteur a pu lui aussi éprouver, ce qui lui permet d'accéder à une vérité non plus sémantique mais représentationnelle : c'est sur ce mécanisme que Dominicy fonde sa théorie de l'évocation (2011 : 148 sq) que j'ai présentée en détail dans Monte 2012.

On voit donc que, du point de vue sémantique, ce poème apparemment simple est extrêmement subtil et complexe : ni l'identité des actants, ni la temporalité ne sont fixées de façon stable, ce qui permet des glissements de l'occurrence au type, du jeu des fillettes au parcours de vie des adultes voulant revenir au pays après avoir longtemps cherché, du temps des personnages à celui du lecteur, qui, lui aussi, se souvient de ces jeux qui faisaient oublier l'arrivée de la nuit et de ce brusque regard aux feuillages devenus sombres. Il est intéressant de noter aussi que les trois scènes rattachées au pays n'ont aucune ambition de complétude : rien sur le travail des adultes mâles, une prédominance 
sinon exclusive («l'enfant» peut référer à un garçon comme à une fille), du moins très forte du féminin, en contraste avec le « ils » initial: la nostalgie du pays serait-elle un désir de retour au monde féminin de l'enfance? L'atmosphère générale, contrairement aux scénarios types où le désir du pays (Heimweh) s'accompagne d'une idéalisation de celui-ci, n'est pas particulièrement euphorique: la vieille femme qui tricote peut être un avatar de la Parque funèbre, la mère est excédée par les cris de son bébé, la place en fin de vers de « brûles » et « noires » peut conduire à interpréter la couleur noire comme l'effet d'une combustion et la vie comme une consumation. Ces possibilités ne sont pas assertées dans le poème, elles sont uniquement suggérées, et soutenues par des faits d'organisation qui relèvent de l'axe II de la textualité.

Le poème semble à première vue moins iconique que le précédent : vers libres variant de 3 à 10 syllabes, texte compact sans strophes ni laisses, pas de rimes. On note cependant que la fin de la première partie s'accompagne d'une récurrence des sonorités nasales introduites par « chemin » (enfant / disant / fin des fins) et que dans la deuxième partie, le son /i/ qui est à la finale de « pays » est mis en valeur par la chaine puis / amie / redit / vie $e^{12}$ et par la répétition de si. On observe aussi que «noires » fait écho à «tard», que les trois adverbes temporels longtemps / tard / bientôt ont une dernière syllabe commençant par / t/ et qu'on peut aussi tracer un fil de «femme » à « feuilles » en passant par « enfant », « fins », « fillettes », fil unissant les âges de la vie sous le signe de la fin, ce qui appuie la vision de la vie comme consumation. Les deux apparitions de la deuxième personne du singulier s'accompagnent d'une mise en valeur du son /y/ par sa place sous l'accent due à l'inversion du pronom au vers 8 et par sa redondance dans «tu brûles », ce qui souligne ce changement énonciatif sur lequel je reviendrai à propos de l'axe III. Sur le plan métrique, on constate que les vers de 3 syllabes associent le « pays » et la « mère ». Le fait que le poème commence par des vers très dissemblables (10-3-7-7-3-6) avant de se stabiliser avec des octosyllabes sauf l'avant-dernier vers (où les tirets remplacent les 2 syllabes en moins) amène à penser que le découpage des vers au début a précisément pour fonction d'isoler et de relier « au pays » et « où la mère ». Bien que l'on ne puisse comparer un sonnet régulier et ce poème, on voit donc

${ }^{12}$ L'antéposition certainement consciente du complément de "redit" "à son amie" est ce qui permet cette mise en valeur du/i/ et l'écho visuel amie/vie. Ce serait très différent si on avait « Puis dans le jeu la fillette / redit à son amie tu brûles » 
que les parallélismes ne sont pas absents ici et soutiennent la production du sens.

Si l'on passe à présent à l'axe énonciatif, on observe tout d'abord un effacement quasi-total du locuteur. Comme le premier poème, celui-ci se donne comme un énoncé non fictionnel, un propos qui pourrait être tenu - nonobstant l'isolement sur la page et le titre - dans une conversation mais le locuteur n'apparait pas, hormis dans la parenthèse énonciative ouverte par les tirets, dont nous avons vu qu'elle joue un rôle capital dans l'aiguillage vers une interprétation métaphorique du jeu des fillettes. Le vocabulaire n'est presque pas subjectif : on ne relève que des évaluatifs non axiologiques ${ }^{13}$ (" un peu », " si longtemps », «si tard») qui situent par rapport à une norme quantitative. Cette volonté d'effacement (Vion 2001, Monte 2007) centre le poème sur les situations évoquées comme si leur évaluation n'était pas en jeu, comme si elle découlait naturellement de ce qui est dit, alors que précisément elle reste problématique en raison du flottement sémantique vu sous l'axe I. Les discours rapportés font l'objet d'un traitement paradoxal : d'une part, ils sont clairement attribués et introduits par des verbes de dire, d'autre part ils ne sont pas signalés par des guillemets. De ce fait « à la fin des fins » peut appartenir aussi bien au discours citant qu'au discours cité, même si le côté familier de l'expression et l'impatience montrée par la répétition de « te tairas-tu » s'accordent plutôt avec la deuxième possibilité. Les deux énoncés sont marqués par l'apparition du $t u$ due à la situation d'interaction, et le /y/ est souligné par sa place sous l'accent dans les deux syntagmes verbe-pronom. L'association phonique des deux situations s'accompagne de subtiles analogies : les deux destinataires n'ont pas vraiment pouvoir sur leur vie. L'enfant qui exprime son désaccord ou son malaise par des cris est sommé de se taire et l'amie suit les indications de la fillette. On peut penser que, pour le locuteur, ces situations résument symboliquement une destinée sur laquelle on a peu de prise. L'évaluation, dans cette perspective, ne se ferait pas directement par des prises de position du locuteur mais indirectement par des choix énonciatifs soulignés structurellement. Ceci nous montre l'interdépendance entre les trois niveaux du texte.

Je propose de synthétiser cette analyse en positionnant le poème de la façon suivante sur les trois axes que j'ai définis :

\footnotetext{
${ }^{13}$ Je me réfère à la typologie proposée par Kerbrat-Orecchioni (1980).
} 


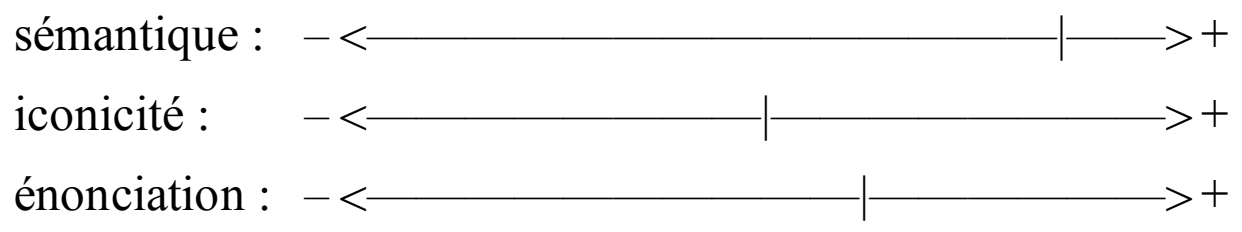

Comme dans le premier poème, quoique de façon différente, la littérarisation de l'énonciation est relativement discrète ; l'iconicité est nettement plus faible, peu perceptible pour un lecteur ordinaire, hormis en ce qui concerne la typographie ; en revanche, la nouveauté de la représentation sémantique ainsi que ses anomalies sont patentes.

\subsection{Un poème en prose}

Yves Bonnefoy, né en 1923, a écrit trois suites de poèmes intitulées Les raisins de Zeuxis, Encore les raisins de Zeuxis et Derniers raisins de Zeuxis, d'abord publiées séparément en livres d'artiste puis rassemblées avec d'autres suites dans La Vie errante (1993). Le poème que je vais étudier est le dernier de la troisième série, il constitue donc la clôture de cet ensemble de 3 fois 9 poèmes en prose. Les auteurs antiques ont rapporté que le tableau de Zeuxis Enfant aux raisins était peint de façon tellement réaliste que les oiseaux venaient picorer la grappe de raisin. Ces poèmes sont donc l'occasion pour Bonnefoy, qui a consacré un livre à la Rome baroque, de réfléchir sur le rapport entre art et réel, entre image et présence. Dans sa bibliographie divisée en 3 sections - poésie, essais, traductions de Shakespeare - , les trois suites figurent dans la section « poésie », mais le fait qu'elles soient en prose va nous amener à réfléchir sur l'éventuelle spécificité de ce type de poèmes.

Du point de vue sémantique, le plus remarquable dans ce texte, c'est le jeu entre le tableau, ce qu'il représente, et celui qui le regarde, jeu qui brouille les frontières entre ces trois ordres de réalité. Le tableau décrit représente, nous dit le texte, une flaque dans laquelle se reflètent des grains de raisins et le « ciel lumineux encore du crépuscule ». La flaque est un « miroir» dans lequel on aperçoit des « ombres claires » et devant elles, d'autres ombres, « noires ». Le texte est donc la description d'un reflet, mais certains fragments du texte - « si l'on s'y penchait on apercevait des ombres de grains », « mais que l'on plonge la main dans le miroir, que l'on remue cette eau » - laissent penser que le spectateur est comme entré dans le tableau, devenu réel : le verbe « se pencher » 
convient bien à une personne au-dessus d'un étang, mais pas au regard sur un tableau, et la main qui agite l'eau n'est pas celle d'un personnage peint mais de celui-là même qui regarde.

Le texte décrit très progressivement le tableau, en distillant les informations par petites touches : « quelque chose comme une flaque », « une flaque », « des ombres claires », «d'autres ombres ». Il ménage la surprise, ne nous disant qu'à la dernière phrase que ces ombres noires sont des oiseaux, mimant ainsi la découverte progressive par l'œil, mais dans un dernier retournement, le tableau s'abolit au profit d'un contact avec une eau réelle qui mêle « l'ombre des oiseaux et celle des fruits ». Par ailleurs, le mot « flaque » fait doublement écho. D'une part, il renvoie à deux autres occurrences, une dans le quatrième poème de la première suite : «j'écris en hâte le mot flaque, le mot étoile » (p.60), l'autre dans le cinquième poème de la deuxième suite où il est question d'un rêve impliquant le peintre Poussin : «j'entrai, il gisait dans des flaques de boue, des gravats, je criai encore « Non, ne meurs pas! » (p.73). On peut penser aussi que le choix du mot, plutôt qu'étang ou même mare, mot volontairement réducteur, trivial, qui plus est modalisé - « quelque chose comme une flaque »-n'est pas sans lien avec la fin du Bateau ivre de Rimbaud : « Si je désire une eau d'Europe, c'est la flache / Noire et froide où vers le crépuscule embaumé / Un enfant accroupi plein de tristesse, lâche / un bateau frêle comme un papillon de mai ». La présence des enfants dans le poème est sans doute dans la droite ligne de cet hommage à Rimbaud, à son choix de la « réalité rugueuse » sur lequel Bonnefoy a beaucoup réfléchi ${ }^{14}$. La flaque se dote ainsi d'une double symbolique, contradictoire : d'une part, elle est dérisoire, prête à sécher, à disparaitre, d'autre part, elle a plus de force et de présence que tous les océans exotiques parcourus par le bateau ivre parce qu'elle est attachée à l'enfance où s'enracine pour Bonnefoy toute la quête poétique future. Notons enfin qu'elle est décrite comme « une brève pensée d'eau brillante, calme », ce qui montre aussitôt que le tableau reconstruit le réel plus qu'il ne l'imite, mais le réel luimême se trouve interrogé. Loin d'être une substance, il est le fruit d'un travail du regard : ce sont les « yeux des enfants » qui isolent « la fantastique découpe » d'une « grappe parmi les pampres ».

${ }^{14}$ Je rappellerai qu'il est l'auteur d'Arthur Rimbaud dans la collection du Seuil Les écrivains d'aujourd'hui. 
La leçon ultime de Zeuxis est une mise en cause du réalisme au profit d'un lieu vide, mystérieux, qui pourrait, en renonçant à copier le réel, en garder la force la plus intense et supprimer la frontière entre l'art et le monde. Telle est du moins mon interprétation de ce poème. En effet, comme dans « Au pays », une scène nous est proposée sans qu'aucun mode d'emploi ne nous soit livré. Le locuteur est beaucoup moins effacé que dans le poème de Follain, puisqu'on relève des adjectifs affectifs (calme, fantastique), et un point de vue très subjectif, qui se manifeste par la modalisation (quelque chose comme, vaguement), l'éventualité (si l'on s'y penchait, que l'on plonge... et), l'établissement d'un contraste entre différents plans, la période très travaillée que constitue la deuxième phrase du premier paragraphe. Les deux premiers on réfèrent à des contemporains de Zeuxis et ne peuvent donc englober le locuteur; les deux derniers peuvent l'inclure, mais il me semble que le texte offre deux points de vue successifs, correspondant aux deux paragraphes : celui du spectateur du tableau et celui du scripteur qui réfléchit sur le tableau en le décrivant. Le texte, malgré son passé simple et ses imparfaits initiaux, s'écarte rapidement des paramètres de la narration ${ }^{15}$ et glisse vers un discours théorique marqué par un présent qui prend une valeur habituelle, sans exclure complètement un ancrage énonciatif amorcé par l'attaque du texte "c'est quelque chose comme » et soutenu par les marques de subjectivité. Le caractère totalement fictif du textesi Zeuxis a véritablement existé, aucun témoignage ne parle de ce tableau d'une flaque - est gommé par le dispositif énonciatif ambigu, de sorte que le texte à la fois exhibe sa littérarité et la masque, dessinant pour le récepteur une place instable, tour à tour lecteur d'une fable dont il est invité à dégager la vérité symbolique et auditeur d'un discours sur la peinture dont il est appelé à valider les assertions.

Le caractère iconique du texte apparait tout d'abord dans les répétitions et les jeux de mots qui font du reflet un des principes d'écriture du texte : le mot « réflexion », vu le contexte, acquiert un double sens, à la fois intellectuel et optique. On remarque que plusieurs mots ou constructions sont répétés deux fois : flaque, ombres, que l'on plonge la main dans le miroir, que l'on remue cette eau. On peut aussi rapprocher « inclinait» et «s'y penchait». Mais ces

${ }^{15}$ Je prends ce mot, de même que celui de « discours théorique » au sens que leur donne Bronckart (1996) dans sa typologie. Voir aussi Adam (2011) pour une analyse similaire des systèmes temporels du français. 
effets de double restent discrets et la dissymétrie des deux paragraphes est flagrante, comme si le scripteur prenait soin d'éviter un trop grand mimétisme entre le référent du texte et sa structure. Le premier paragraphe obéit à une syntaxe de l'expansion vers la droite, par ajouts apparemment non prémédités : la phrase 1 offre la structure rhème - thème, au lieu de la structure canonique sujet - groupe verbal. La seconde reprend le rhème « une flaque » en le précisant par une longue apposition, puis, dans la partie coordonnée par et, propose une série de groupes amples qui expansent le complément d' " apercevait » à partir du rattachement lâche d'un SN grâce à la préposition « avec ». Le découpage syntaxique aboutit à un alexandrin : $6-10-10-7-7-7-12$. Le second paragraphe, au contraire, repose sur le rétrécissement après un complément initial plus ample : la phrase 3 a un rythme 6 - 3-4, et la phrase 4 un rythme 11-6-112 avec un verbe à complément pronominal antéposé qui clôt brusquement une phrase dotée d'un long cadre et d'un long groupe sujet. Il semble qu'au plaisir de la phrase nombreuse associé à des souvenirs d'enfance et à la lumière adoucie du crépuscule, succède un paragraphe plus austère où le rythme oblige à se déprendre. On peut y voir une figure du cheminement de Zeuxis tel qu'il est conté dans les neuf poèmes de cette suite : d'abord gêné par les oiseaux qui lacèrent ses tableaux, il constate un jour avec soulagement qu' ils les délaissent, puis il s'en afflige:

Ah, que s'est-il passé, se demande-t-il ? A-t-il perdu le sens de ce que c'est que l'aspect d'un fruit, ou ne sait-il plus désirer ou vivre ? (début du poème 7)

Il revient finalement à ses pinceaux pour peindre non plus des raisins mais une flaque où ils se reflètent et où se résout la contradiction entre la beauté et la violence du monde. La cadence de la dernière phrase, imposant un retrait, un vide, est peut-être là pour nous indiquer qu'un tel choix va contre nos habitudes, nos tentations. Il y aurait donc bien iconicité, mais non pas du côté du redoublement du reflet, mais au contraire dans la déprise, le dégrisement à quoi nous oblige la dernière phrase.

Je proposerai de synthétiser cette analyse du texte par le schéma suivant :

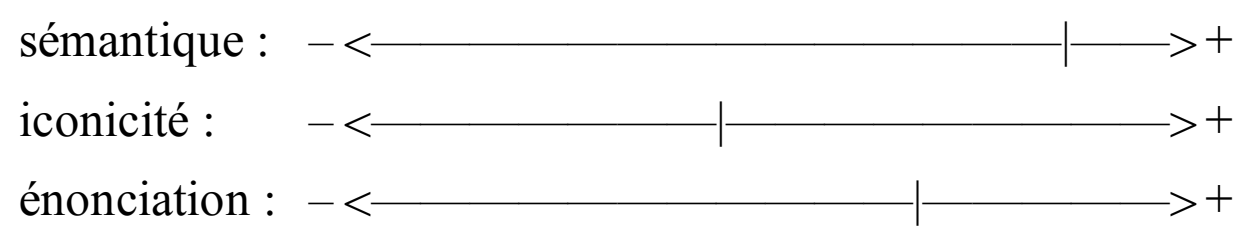


MONTE, M. Pour une conception historicisée...

Il me semble en effet que la représentation sémantique du poème est complexe et éloignée de nos habitudes, que son iconicité, quoique masquée par la prose, n'est pas négligeable et que son dispositif énonciatif est suffisamment instable pour nous alerter sur la dimension littéraire du texte et pour requérir du lecteur une réception spécifique.

\subsection{Comparaison}

Compte tenu de leur période historique et des choix de leurs scripteurs, ces trois poèmes ne sont pas poétiques de la même façon. Une enquête auprès de locuteurs ordinaires le montrerait aisément. En proposant trois axes différents pour observer les textes, je peux rendre compte facilement de ce phénomène sans renoncer à appeler poèmes ces trois textes, appellation revendiquée par leurs auteurs et qui dit quelque chose de leur positionnement dans le champ littéraire.

L'axe sémantique a trait à la nouveauté de la représentation discursive construite par le texte. Dans les siècles passés, la recherche des poètes visait à proposer une représentation frappante mais pas nécessairement incongrue ou nouvelle. Les Méditations de Lamartine, par exemple, ou Les Contemplations de Victor Hugo, comme les sonnets de Sponde, occupent sur cet axe une position moyenne. C'est à partir de la fin du XIXe siècle que les poètes légitiment leur position littéraire par cette capacité d'invention sémantique, que Rimbaud illustre avec éclat et qui devient peu ou prou un des axes majeurs de la poésie du XXe siècle. Même si certains poètes, comme Follain, revendiquent une poésie du quotidien, une poésie du peu (Viart 1998: 60) et se tiennent à l'écart des recherches surréalistes qui placeraient, elles, les textes qui en sont issus à l'extrémité positive de l'axe sémantique, une observation fine de leurs poèmes montre néanmoins des décalages constants par rapport aux façons habituelles de dire.

L'axe iconique a trait au fait que le texte exhibe ou non son travail spécifique sur le matériau verbal et s'appuie sur ce matériau pour produire des effets de sens et suggérer des interprétations. Les poèmes à forme fixe comme les sonnets, et plus généralement les poèmes à contraintes, se situent à l'extrémité positive de cet axe ; les poèmes en vers libres s'affichent encore comme poèmes par le choix du vers, ce qui implique une verticalité qui s'oppose à l'ordre linéaire 
de la prose, et des interruptions qui ne correspondent pas forcément à des pauses syntaxiques. Mais l'iconicité passe aussi par des récurrences phoniques et des phénomènes accentuels ou rythmiques que l'on peut trouver dans la prose et qui peuvent en revanche faire défaut dans des poèmes écrits en "vers de mirliton " où l'habillage du vers ne masque pas l'absence de récurrences phoniques et de parallélismes syntactico-rythmiques créateurs de sens. L'iconicité implique en effet une alliance entre le niveau du matériau phonique et graphique et le niveau sémantique, alliance qui fait passer le sens par d'autres canaux que le simple agencement des propositions.

Du point de vue énonciatif, les poèmes de Sponde et Follain occupent des positions moyennes; ils peuvent apparaitre de prime abord comme des énoncés ordinaires: celui de Sponde s'apparente à une prédication, celui de Follain à un récit fait à des proches à propos de gens connus (d'où le « ils » initial). Mais cette apparence est mise en question, non seulement par l'appartenance affichée à l'archi-genre poésie mais aussi par une série de dérèglements au fil du poème qui viennent confirmer la nature seconde - au sens de Bakhtine - de ces textes. Montrant leur énonciation, ils amènent le lecteur à s'interroger sur les conditions dans lesquels ils sont énoncés et à conceptualiser l'écart entre le locuteur construit par l'énoncé et un sujet empirique. La mise en scène énonciative est un peu plus marquée dans le poème de Bonnefoy en raison de l'abandon de la narration pour un discours au présent qui hésite entre ancrage et désancrage mais qui abolit la distance temporelle initialement posée. Comme je l'ai dit plus haut, ces trois poèmes présentent des traits énonciatifs typiques de la poésie dite lyrique (Monte 2003) qui combine généralement des traits du discours interactif ordinaire et une décontextualisation qu'elle partage avec un certain nombre de formes courtes (proverbes, devinettes, petites histoires, etc.) et qui contribue à sa littérarité. Il n'est pas sûr que la poésie satirique ou la poésie dite de circonstance sur lesquelles je n'ai pas travaillé partagent exactement les mêmes traits et il est certain que la poésie épique ou les fables s'en différencient par leur fictionalité : le décrochage par rapport au monde ordinaire y est plus apparent, plus assumé, et le rôle dévolu au lecteur est celui d'un auditeur invité à jouir du récit mais aussi à en tirer des leçons et non celui d'un coénonciateur amené à entrer dans l'espace énonciatif du poème et à en valider l'orientation illocutoire. Sur cet axe, les différences relèvent moins, comme pour les axes précédents, de l'évolution historique de la poésie que de subdivisions à l'intérieur de l'archi-genre dès les premiers textes dont nous disposons. 


\section{Conclusion}

Pour conclure, je dirai qu'un tel modèle, même s'il est encore à affiner, permet de penser la poéticité de façon plus adéquate qu'une opposition non opérante entre langage ordinaire et langage poétique ou même que la notion trop englobante de fonction poétique qui ne permet pas, dans les écrits de Jakobson (1963), de différencier un texte poétique et un texte publicitaire. La prise en compte de trois axes partiellement indépendants, d'une part, suggère des rapprochements qu'on n'aurait peut-être pas imaginés (entre les poèmes et les textes scientifiques, par exemple, ou entre les poèmes et les petites histoires), d'autre part, montre que l'ajout à la structure logico-sémantique de parallélismes phoniques, rythmiques, métriques, syntaxiques, est essentiel à la poésie, mais ne suffit pas à la caractériser. Les dimensions sémantique et énonciative jouent un rôle crucial dans le fonctionnement global d'un texte et, jointes à la dimension iconique, permettent à l'intérieur de l'archi-genre poésie une caractérisation plus fine des poèmes qui tient compte de leur moment historique et de leur genre spécifique. Enfin, le modèle rend possible un jugement graduel de poéticité qu'il sera intéressant de confronter aux perceptions des lecteurs ordinaires et aux jugements des critiques.

\section{Bibliographie}

ADAM, J.-M. (2éd. revue). La Linguistique textuelle. Paris: Armand Colin, 2011. AMOSSY, R. L'argumentation dans le discours. Paris: Nathan, 2000. (nouvelle édition 2006, Armand Colin)

BARBÉRIS, J.-M. Subjectivité dans le langage. In: DÉTRIE, C., SIBLOT, P. \& VERINE, B. (éd.), Termes et concepts pour l'analyse du discours. Paris: Champion, 2001.

. Subjectivité en même vs en soi-même. In: DÉTRIE, C., SIBLOT, P. \& VERINE, B. (éd.), Termes et concepts pour l'analyse du discours. Paris: Champion, 2001.

BONHOMME, M. Pragmatique des figures du discours. Paris: Champion, 2005.

BRONCKART, J.-P. Activité langagière, textes et discours. Paris-Lausanne: Delachaux et Niestlé, 1996.

CULIOLI, A. Pour une linguistique de l'énonciation, tome3, Paris-Gap: Ophrys, 1999. 
DOMINICY, M. Poétique de l'évocation. Paris: Garnier, 2011.

DUCROT, O. Le dire et le dit. Paris: Minuit, 1984.

FAVRIAUD, M. Plurisystème ponctuationnel, dimension, intensité des signes et architecturation du texte poétique. In Langue française 172, 2011, p. 83-98.

GENETTE, G. Fiction et diction. Paris: Seuil, 1991.

GRIZE, B. Logique et langage. Gap: Ophrys, 1999.

HAMBURGER, K. Logique des genres littéraires. Trad.fr. (2 édition révisée en allemand : 1968). Paris: Seuil, 1986.

KERBRAT-ORECCHIONI, C. L'Énonciation. De la subjectivité dans le langage. Paris: Armand Colin, 1980.

JAKOBSON, R. Essais de linguistique générale I : Les Fondations du langage. (trad.fr.), Paris: Minuit, 1963.

. À la recherche de l'essence du langage. Traduit de l'anglais par J. Havet, dans Problèmes du langage. Paris: Gallimard (Diogène $\mathrm{n}^{\circ}$ 51), 1966. p. 22-38.

LEJEUNE, P. Le pacte autobiographique. Paris: Seuil, 1975.

MAINGUENEAU, D. Analyser les textes de communication. ( $2^{\mathrm{e}}$ éd. revue et augmentée). Paris: Armand Colin, 2007.

MOIRAND, S. Les discours de la presse quotidienne: Observer, analyser, comprendre. Paris: PUF, 2007.

MONTE, M. Essai de définition d'une énonciation lyrique. L'exemple de Philippe Jaccottet. Poétique, n 134, p.159-181, 2003.

. Variable élégie. Dans Elégies, Babel n ${ }^{\circ} 12$, revue de la Faculté de Lettres de l'Université de Toulon et du Var, p.7-12, 2005.

. Poésie et effacement énonciatif. Semen 24. Besançon: Presses Universitaires de Franche-Comté, 2007. p. 99-121.

. Pour une autonomie relative des niveaux sémantique, iconique et énonciatif des textes poétiques. Actes du Congrès Mondial de Linguistique Française, à paraitre prochainement sur le site de l'ILF, 2012.

. Pour une conception historicisée et graduelle de la poéticité L'interaction des dimensions sémantique, énonciative et iconique dans trois poèmes (Sponde, Follain, Bonnefoy). In. HRUBARU, Florica; POP, Liana (Eds.). Revue Recherches: Langue et discours - Actes du XVIIIe Séminaire de Didactique Universitaire Constanta 2011. Cluj: Echinox, 2012, pp. 14-39 
RASTIER, F. Sémantique interprétative. Paris: PUF, 1987.

RUWET, N. Parallélismes et déviations en poésie. In: KRISTEVA, J. et al. (éds). Langue, discours, société. Paris: Seuil, 1975. p. 307-351.

VIART, D. Moments du sujet. Littérature nº198, p. 49-60, 1998.

VION, Robert. Effacement énonciatif et stratégies discursives. In: JOLY, A. \& DE MATTIA, M. (éds.). De la syntaxe à la narratologie énonciative. Paris: Ophrys, 2001.p. 331-354.

\section{Annexes}

Poème de Jean de Sponde (Essai de quelques poèmes chrétiens, 1588)

Tout le monde se plaint de la cruelle envie

Que la nature porte aux longueurs de nos jours :

Hommes, vous vous trompez, ils ne sont pas trop cours,

Si vous vous mesurez au pied de vostre vie.

Mais quoy? je n'entens point quelqu'un de vous qui die :

Je me veux despestrer de ces fascheux destours, Il faut que je revole à ces plus beaux sejours, Où sejourne des Temps l'entresuitte infinie.

Beaux sejours, loin de l'oeil, pres de l'entendement, Au prix de qui ce Temps ne monte qu'un moment, Au prix de qui le jour est un ombrage sombre,

Vous estes mon desir : et ce jour, et ce Temps, Où le Monde s'aveugle et prend son passetemps, $\mathrm{Ne}$ me seront jamais qu'un moment et qu'une Ombre. 
Poème de Jean Follain (Territoires 1953)

Au pays

Ils avaient décidé de s'en aller

au pays

où la même vieille femme

tricote sur le chemin

où la mère

secoue un peu l'enfant

lui disant à la fin des fins

te tairas-tu, te tairas-tu?

Puis dans le jeu à son amie

la fillette redit tu brûles

et l'autre cherche si longtemps

si tard - ô longue vie -

que bientôt les feuilles sont noires.

Poème d'Yves Bonnefoy (Derniers raisins de Zeuxis, 1998)

C'est quelque chose comme une flaque, le dernier tableau que Zeuxis peignit, après longue réflexion, quand déjà il inclinait vers la mort. Une flaque, une brève pensée d'eau brillante, calme, et si l'on s'y penchait on apercevait des ombres de grains, avec à leur bord vaguement doré la fantastique découpe qui ourle aux yeux des enfants la grappe parmi les pampres, sur le ciel lumineux encore du crépuscule.

Devant ces ombres claires d'autres ombres, celles-ci noires. Mais que l'on plonge la main dans le miroir, que l'on remue cette eau, et l'ombre des oiseaux et celle des fruits se mêlent.

Recebido: 11/10/2013

Aprovado: 08/11/2013 